\title{
The Study of Crew Physical and Psychological Evaluation Model and Sports Intervention Method
}

\author{
Guo-Qing Li*, Bin Zhang \\ Department of Physical Education, Harbin Engineering University, Harbin, 150001, China. \\ Received 13 June 2015; received in revised form 27 July 2015; accepted 01 August 2015
}

\begin{abstract}
It is very important to research the physical and psychology of crew for ship safe. The artificial neural network theory is applied to the crew health evaluation, according to the crew health evaluation index. The corresponding BP neural network for health state evaluation established. Training samples of neural network obtained using the expert experience and statis tical data. With the aid of computer and MATLAB software, the evaluation results can be conveniently achieved. BP neural network model that was trained can be operated "off-line", evaluate the crew physical and psychological health status accurately and effectively. And the guidance and recommendations of sports items intervention measures were given by crew health expert system. All these research will help the crew to improve the level of physical and psychology health.
\end{abstract}

Keywords: physical and psychological evaluation, sports intervention, BP neural network

\section{Introduction}

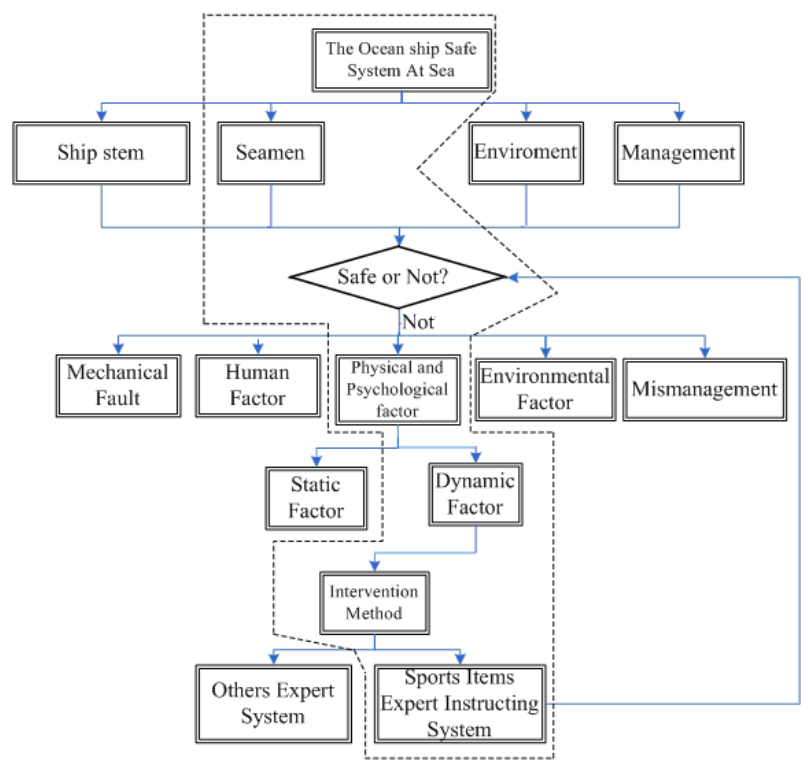

Fig. 1 Block diagram range of study

Ocean shipping has the characteristics of drifting time longer at sea, away from land and shore. So the crew's physiological status, psychological status and level of technology have put forward higher requirements. It has been shown that human misplay is the main cause of ship accident factors from the large number of maritime case analys is at intemal and abroad. Therefore, it is of great significance to analyze and research the physical and psychological status. Which can take the corresponding methods and preventive measures to reduce the dynamic human misplay factors, as we known these factors would cause ship mechanical accident. The research of human factors and ergonomics is much, but it is not much to achieve the practical application. Especially the establishment of crew physical and psychological health evaluation model is rarely. This paper will made crew physical and psychological health evaluation model for ocean shipping at sea eventually, to track and evaluate crew health status, take intervention measures, reduce human accidents. System frame as shown in Fig. 1. The research field of this paper is in the lengthways range of dashed.

\section{Theory of BP Neural Network}

BP neural network trained the network using back propagation method, and then network weights can be got. The main idea is modify the weight or threshold to make the error function decline along the negative gradient direction. Signal transmission and error back-propagation are two process procedures of BP neural network leaming. In the forward propagation, the signal is inputted from input layer, and propagated to output layer after processed by hidden layer. Each neuron only could affect the neurons state of the next layer. The error propagates back if desired output has not been got. Output error which is the basis to modify the neurons weight back propagate to input layer through hidden layer, repeat until output error permitted[1-3].

Essentially, BP neural network is a nonlinear optimization problem issues for a set of input and output samples. It can be seen as a mapping from $\mathrm{n}$-dimensional to m-dimensional [4]. BP neural network structure is shown in Fig. 2.

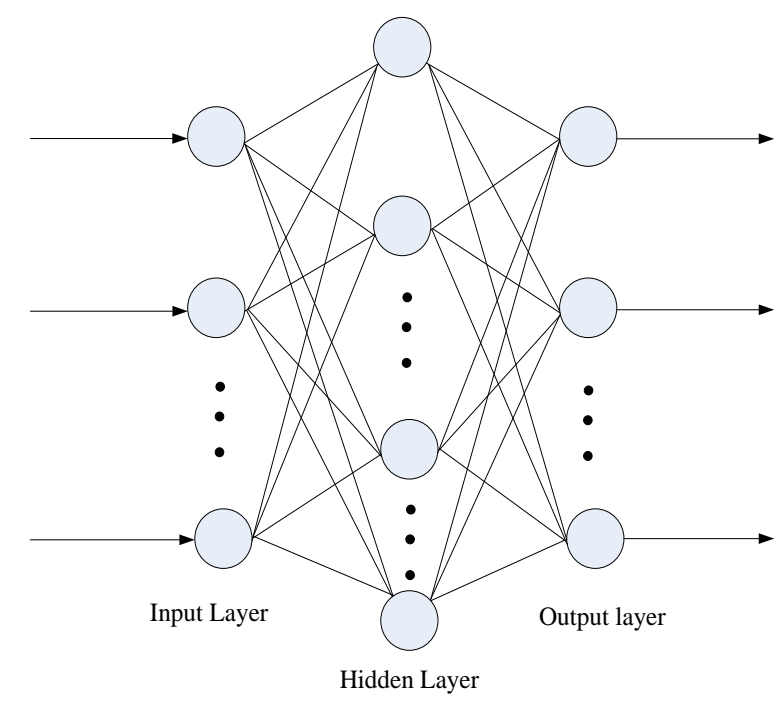

Fig. 2 Structure of neural network 


\section{Crew Physical And Psychological Health Evaluation Model}

Definition: the research is limited in the dynamic factors of the ocean crew physical and psychological health evaluation index, variable factors, adjustable factors, controllable factors)

\subsection{Index of Physical and Psychological Health}

The key research question of this paper is to abstract characteristic variable that can reflect the state of physical and psychological from the index of crew physical and psychological health. However it is gratified for researchers that a series of index can be adjusted for model and as the application of the model, the extraction index of evaluation system will be closer to the crew physical and psychological state. Embodied in the BP neural network model structure is to use the model as simple as possible to evaluate accurately the crew physical and mentalhealth status.

\subsection{The Modeling of Crew Physical and Psychological Health}

The theory has been proven three layer forward feedback neural network can approximate arbitrary continuous function with arbitrary accuracy. The BP neural network of this paper is three layer structures [5-6]. The input signal is move the spread to the hidden layer nodes, through the transfer function then output signal of hidden layer nodes is trans mitted to the output layer. Trans fer function from the input layer to the hidden layer and hidden layer to the output layer used the Tansig function and Purelin function.

The theory has been proven three layer forward feedback neural network can approximate arbitrary continuous function with arbitrary accuracy. The BP neural network of this paper is three layer structures. The input signal is move the spread to the hidden layer nodes, through the transfer function then output signal of hidden layer nodes is trans mitted to the output layer. Transfer function from the input layer to the hidden layer and hidden layer to the output layer used the Tansig function and Purelin function.

The ocean crew physical and psychological health at sea evaluation model chose the index of fatigue, sleep, appetite, food egestion, loneliness, anxiety, depression, panic, emotionality tension, thinking ability, self-control ability as the input of BP neural network, and crew health level, health, sub-health, unhealthy, serious unhealthy as output. The number of hidden nodes depends on many factors such as the quantity of training sample and the rule complexity of sample contains. According to experience hidden nodes is 8 . Some of the variables results of the physical and psychological health were chosen as BP neural network training set. The training samples are shown in Table 1.

Table 1 The training sample set of BP neural network

\begin{tabular}{|c|c|c|c|c|c|c|c|c|c|c|c|}
\hline & M1 & M2 & M3 & M4 & M5 & M6 & M7 & M8 & M9 & M10 & M11 \\
\hline $\mathrm{d} 1$ & 0.20 & 0.05 & 0.10 & 0.10 & 0.10 & 0.00 & 0.05 & 0.00 & 0.00 & 0.00 & 0.00 \\
\hline $\mathrm{d} 1$ & 0.20 & 0.05 & 0.05 & 0.05 & 0.05 & 0.00 & 0.05 & 0.00 & 0.00 & 0.05 & 0.00 \\
\hline $\mathrm{d} 1$ & 0.00 & 0.10 & 0.00 & 0.00 & 0.00 & 0.10 & 0.00 & 0.05 & 0.00 & 0.10 & 0.00 \\
\hline $\mathrm{d} 2$ & 0.10 & 0.20 & 0.00 & 0.00 & 0.50 & 0.20 & 0.20 & 0.00 & 0.00 & 0.00 & 0.00 \\
\hline $\mathrm{d} 2$ & 0.00 & 0.05 & 0.35 & 0.10 & 0.30 & 0.50 & 0.20 & 0.20 & 0.10 & 0.10 & 0.10 \\
\hline $\mathrm{d} 2$ & 0.00 & 0.10 & 0.00 & 0.00 & 0.00 & 0.20 & 0.10 & 0.20 & 0.15 & 0.00 & 0.20 \\
\hline $\mathrm{d} 3$ & 0.20 & 0.75 & 0.10 & 0.10 & 0.10 & 0.30 & 0.00 & 0.00 & 0.30 & 0.20 & 0.20 \\
\hline $\mathrm{d} 3$ & 0.60 & 0.05 & 0.05 & 0.05 & 0.25 & 0.15 & 0.15 & 0.60 & 0.80 & 0.25 & 0.40 \\
\hline $\mathrm{d} 3$ & 0.25 & 0.10 & 0.00 & 0.00 & 0.60 & 0.10 & 0.05 & 0.05 & 0.30 & 0.10 & 0.20 \\
\hline $\mathrm{d} 4$ & 0.00 & 0.60 & 0.50 & 0.50 & 0.60 & 0.10 & 0.10 & 0.10 & 0.10 & 0.30 & 0.40 \\
\hline $\mathrm{d} 4$ & 0.90 & 0.30 & 0.40 & 0.30 & 0.10 & 0.70 & 0.15 & 0.25 & 0.60 & 0.20 & 0.20 \\
\hline $\mathrm{d} 4$ & 0.60 & 0.60 & 0.05 & 0.05 & 0.00 & 0.15 & 0.15 & 0.75 & 0.10 & 0.35 & 0.40 \\
\hline
\end{tabular}

D-health level, D1- health, D2- sub-health, D3- unhealthy, D4- seriously unhealthy, M- index of health status, M1- fatigue, M2- sleep, M3- appetite, M4- food egestion, M5- lonely, M6- an xiety, M7- depression, M8- panic, M9-emotional tension, M10- thinking ability, M11- self-control ability

Since centralized degree of the sample data is high, the data is processed by normalization. Every feature is in the 0-1 interval. The interval value indicated the degree, for example: if the sleep state is good, then the value is recorded 0.00 , insomnia recorded 1.00. The state of sleep quality drop a little, but can be a good rest, recovery is perfect, recorded 0.60 . Sleep quality drops badly, the recovery is bad, recorded 0.40. MATLAB software is used to train sample set.

\subsection{The Modeling of Crew Physical and Psychological Health}

After network training, the test will be carried on. The purpose of network test is to see whether the network has good generalization ability. Select 4 set of data as the network testing input, the testresults are shown in Fig. 4.
From Fig. 3-4 we can see, the generalization ability of the network is good, you can use the following BP neural network for ocean crew to evaluate health state.

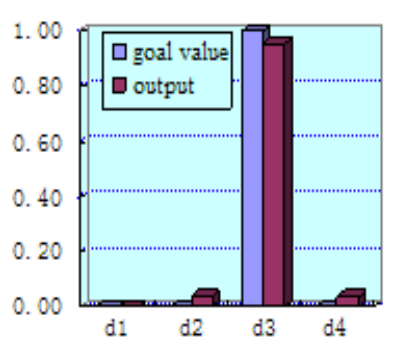

Fig. 3 Output of No. 1 test sample

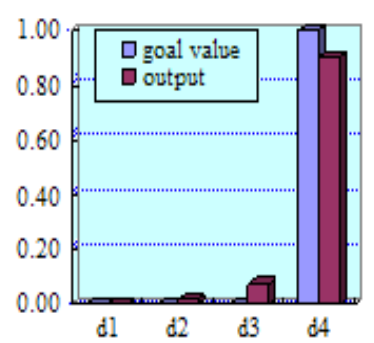

Fig. 4 Output of No. 2 test sample 
Table 2 The test sample of network

\begin{tabular}{|c|c|c|c|c|c|c|c|c|c|c|c|}
\hline No. & M1 & M2 & M3 & M4 & M5 & M6 & M7 & M8 & M9 & M10 & M11 \\
\hline 1 & 0.15 & 0.00 & 0.00 & 0.00 & 0.60 & 0.35 & 0.40 & 0.00 & 0.10 & 0.10 & 0.10 \\
\hline 2 & 0.80 & 0.70 & 0.10 & 0.10 & 0.30 & 0.30 & 0.50 & 0.10 & 0.25 & 0.20 & 0.20 \\
\hline
\end{tabular}

\section{The Sports Items Intervention Expert System}

The crew should strengthen the physical exercise, learn to relax themselves. According to the ship characteristic, it is beneficial to health to adjust measures to local conditions to carry out some physical exercise and a proper amount of entertain ment activities. The health will be improved.Working state is energetic. The ability that copes with stress will be enhanced. The crew relaxed to control themselves when they meet pressure and feet nervous. All these mainly need the crew own conscious physical training in different ways.

The crew sports consciousness is weakness, some of them do not grasp the scientific physical exercise knowledge and methods, have no phys ical exercise habits. And the work burden is heavier during navigation, physical and mental fatigue, sports inert is larger, the necessary physical guidance and supervisory mechanism is not perfect. This research will eventually build physical and psychological health state expert system of ocean crew sports intervention to help them providing references and suggestions that how to choose sports items at sea.

\subsection{Sports project selection principle of crew health state intervention}

According to the physical and psychological health state as mentioned above, this paper put forward the exercise treatment, chose several easy to carry out on board. These sports have better effect on common physical and psychological illness. Brief introduction is as followed [4-6].

\section{(1) Tai Chi}

Body joints, muscles and bones will each pull, press and be diastolic, when playing Tai Chi. Massage effect will be in Visceral due to abdominal breathing, and it is beneficial for intestinal peristalsis with increasing of diaphragmatic upper and lower amplitude. It is beneficial for them to practice Tai Chi at sunrise or sunset on the spacious deck in the vast sea. Tai Chi can train the crew to be happy and pleased with them, and be in a calm mood.

\section{(2) Ear action practice}

The helix covers the ears when doing exercise, rub the earhole with the root of palm (i.e. massage earhole that be covered by helix), Rub to the ear feel itch, when the blood circulate to the ear. It is help to relief tinnitus.

\section{(3) Sit-ups}

This sports can exercise the leg strength also has very good effect on the waist, of course you can use the squat method.

\section{(4) Barbell practice}

Carry barbell with shoulder, helps to improve renal function, also can achieve the effect of alleviating tinnitus.
(5) Walking on the deck

Walking exercise method is very simple, because the tonnage of ocean shipping is very large. The broad deck area is equivalent to two football fields. The crew can make full use of the convenient conditions, when the rising in every morning the crew can enjoy the beauty on the sea and walk or jog on the open deck. This sport not only may increase the cardiopulmonary function, but also can cultivate their original nature, condition psychology. This sports mode has good effect on rehabilitation and prevention of bronchitis for crew.

\section{(6) Climbing gangway ladder}

A certain number of gangways are in ship limited area. The crew can enhance their ability of respiratory system by climbing the gangway ladder coordinating with the movement of breath training. First suction with the nose, then narrow lips to breathe out, each climb2 steps breathe again, this sport can be realized on every ship.

\section{(7) Comfortable slow motion in cabin}

The crew work on board for long-term, often climb cabin, so is very tired. In e course of time they will be facing lumbago and skelalgia, which cannot relieve by energetic exercise, but massage, and do moderate exercise. For deck narrow, poor conditions of the ship, the crew can take Comfortable and slow motion in cabin to for rehabilitation. The concrete forms can reference interface of expert system. Please see Figs. 6-7.

\subsection{The Interface of Crew Health Evaluation Model and Sports Items Intervention Expert System}

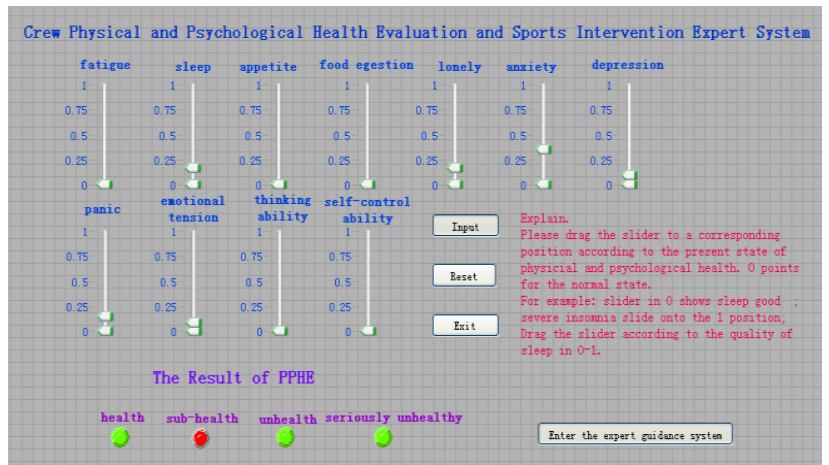

Fig. 6 The interface of crew health evaluation model

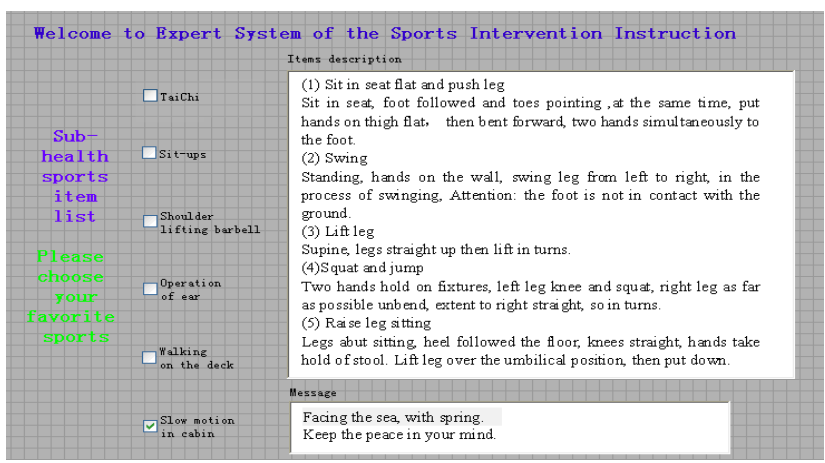

Fig. 7 The interface of s ports items intervention expert system 


\section{Conclusions}

The crew health evaluation model based on BP network and the sports items intervention expert system established in the paper. The system can track and guide $n$ the crew physical and psychological health status at sea in real time. The research work has important practical significance and great practical value, which may enhance the crew physical and psychological health, improve work efficiency, promote the harmony and stability of the ship small environment, maintain the normal life order and ensure safety at sea.

China ocean ships have no crew physical and psychological evaluation system in using at present. Although training data is limited to the present study training model, this study software is carried in ship will have a large number of training data, and classification ability of the network will be constantly improved.

\section{References}

[1] L. C. Jiao, Neural network calculation, Xi'an Xidian Univer sity press, 1996. (In Chinese)

[2] C. R. Yuan, Artificial neural networks and its application, Bei Jing Tsinghua University press, 1999. (In Chinese)

[3] C. D. Xing, J. M. Yang, Q. S. Ren, Theory of artificial intelligent and its application, Shang Hai Dong Hua University press, 2005. (In Chinese)

[4] M. A. Gonzalez, "An integrated logistics support system for training crew medical officers in advanced cardiac life support," Ph.D. Dis sertation, Dept. Industrial Engineering, Cullen College of Engineering, Houston Univ., Houston, TX, 1995.

[5] G. Q. Li, "Discuss effect of physical exercise on the positive role of sub health," Journal of Yichun University, vol. 32, no. 12, 2010.

[6] M. Q. Chen, F. Y. Qiao, "Research on sports effect of occupation disease prevention and treatment for crew," Science and Technology Innovation Herald, no. 35, 2011. (In Chinese) 